\title{
CEuvrer pour le « non pas »
}

\section{Pierre Parlant}

\section{(2) OpenEdition}

Journals

Édition électronique

URL : http://journals.openedition.org/ccs/1891

DOI : $10.4000 /$ ccs. 1891

ISSN : 2558-782X

\section{Éditeur :}

Presses universitaires de Rennes, Association des lecteurs de Claude Simon

\section{Édition imprimée}

Date de publication : 30 août 2018

Pagination : 233-241

ISBN : $978-2-7535-7489-2$

ISSN : 1774-9425

\section{Référence électronique}

Pierre Parlant, « Euvrer pour le «non pas » », Cahiers Claude Simon [En ligne], 13 | 2018, mis en ligne le 30 août 2019, consulté le 20 février 2020. URL : http://journals.openedition.org/ccs/1891 ; DOI :

10.4000/ccs. 1891 


\title{
CEUVRER POUR LE « NON PAS »
}

\author{
Pierre PARLANT ${ }^{1}$
}

"Non pas "une" chaise, "une" table, "un" bol, mais "la" chaise, "la" table, "le" bol. "

Claude Simon, Le Vent

Il y a d'abord, comme s'il s'agissait d'une prescription chez les photographes, le fait de ne jamais donner à voir, lors du tirage, autre chose que ce qui procède de la prise de vue elle-même. Prescription cardinale dont le principe, paraphrasant la maxime en vigueur à l'instant de témoigner, se formulerait ainsi : faire une photo, c'est se vouloir et se déclarer fidèle à ce qu'on a vu, à tout ce qu'on aura vu, à cela seulement.

Au détour d'une phrase, il y aurait ensuite un aveu, une sorte de contrepoint à ladite prescription, aussi serein qu'assuré, celui de l'écrivain, photographe à ses heures:

il m'arrive le plus souvent (parfois aussi faute de disposer d'un téléobjectif) de cadrer mon " sujet » très largement, sachant qu'au calme de la chambre noire, sans hâte et en toute tranquillité, je pourrai toujours supprimer ce qui me semble inutile, risque de disperser l'attention ou de déséquilibrer la composition ${ }^{2}$.

Aveu auquel Claude Simon s'empresse d'ajouter, histoire d'être quitte, la concession suivante: "Il se peut que les professionnels blâment ou méprisent une telle façon de faire, mais je n'ai pas leur "métier" ${ }^{3}$."

1. Pierre Parlant est écrivain, auteur de prose non romanesque et de poésie. Il consacre également des articles à des écrivains contemporains. Dernier ouvrage paru: Ma durée Pontormo (Nous, coll. "Via ", 2017).

2. C. Simon, Photographies, Maeght Éditeur, Paris, 1992, p. 16.

3. Ibid. 
Ne pas "avoir le métier ", revendiquer sans fausse honte le statut d'amateur ${ }^{4}$, telle serait donc l'explication d'une manière peu orthodoxe de pratiquer la photographie. Celle, en somme, qui ferait agir comme on peut, selon un désir certes authentique, mais toujours avec les moyens dont on dispose, c'est-àdire très souvent par défaut. Retravailler le cadrage compenserait la maladresse.

On s'en contenterait. On le ferait d'autant mieux en se souvenant de ce qu'un homme, justement du "métier ", avait écrit à ce propos:

On doit situer son appareil dans l'espace par rapport à l'objet, et là commence le grand domaine de la composition. La photographie est pour moi la reconnaissance dans la réalité d'un rythme de surfaces, de lignes ou de valeurs; l'œil découpe le sujet et l'appareil n'a qu'à faire son travail, qui est d'imprimer sur la pellicule la décision de l'œil ${ }^{5}$.

Photographier serait donc ça, assez peu à vrai dire s'il n'y avait précisément cette aptitude à reconnaître, au niveau le plus immédiat de la "réalité » telle qu'elle se donne à nous, un sens constitué au regard d'une architectonique furtive dont le chiffre est le "rythme », l'outil faisant le reste. Admettons. À condition toutefois de tenir pour acquis le crédit accordé au doigt et à l'œil du photographe, lequel ayant saisi - ou cru voir - ici et maintenant quelque chose, appuie sur le déclencheur en toute connaissance de cause. À condition aussi de considérer la réalité en question dépositaire d'un sens toujours déjà là ; un sens qui lui soit aussi homogène qu'il s'avère porteur d'intelligibilité. Or cela reste à voir. Comment peut-on être sûr qu’à ce geste, tout expérimenté et sincère qu'il se prétende, corresponde une authentique " décision ", assortie de surcroît d'une " reconnaissance » de quoi que ce soit? Comment en outre garantir que la fraction infime de ce temps pendant lequel s'écarte l'obturateur ouvre sur cet «instant décisif» cher à Cartier-Bresson? Quelles que soient les réussites indiscutables qui en résultent - lesquelles occultent au passage la somme des ratages -, ce parti-pris ne relève-t-il pas d'un idéal de maîtrise plus que d'une quelconque positivité?

Si l'on s'en tient à la position de Claude Simon, on ne contestera pas qu'existe en tout cas un départ, sinon un différend, entre ce que font les photographes - les " professionnels » - et ce que, muni de son appareil puis dans la solitude appliquée de la chambre noire, lui-même aura longtemps expérimenté. À bien y regarder, il est d'ailleurs probable que ce différend tienne moins à une expertise présumée chez eux et manquante chez lui, qu'il n'engage en réalité la

4. On pense évidemment ici à l'Album d'un amateur, Remagen-Rolandseck, Rommerskirchen Verlag, 1988.

5. H. Cartier-Bresson, L'Instant décisif, préface à Images à la sauvette, Verve, 1952. 
conception de ce qu'est un "sujet » en photographie. Ce n'est pas rien. S'esquisse alors en effet, à des degrés divers, une esthétique et même une éthique du voir. On en trouve du reste l'intuition, énoncée comme en creux, dans un passage d'Histoire où il est fait mention de l'acte de photographier. Claude Simon y récuse l'idée triviale selon laquelle la photographie est " un de ces instantanés, une de ces coupes lamelliformes pratiquées à l’intérieur de la durée ${ }^{6}$ ". Façon de rappeler que le temps inhérent à l'image - temps d'une narration en puissance - n'est ni identique ni équivalent à celui du cliché. Qu'une prise de vue suppose un "laps ", comme l'a vu et pensé Denis Roche, est une vérité à partir de laquelle rien ne permet d'affirmer que la photographie elle-même, à travers ce qu'elle expose ou retient - l' "ellipse ", du même Denis Roche -, se réduise à un prélèvement, à une " coupe " mécanique effectuée dans la durée. Lorsque Cartier-Bresson s'autorise à parler d'images à la sauvette, de photos prises à la dérobée, dépendant chaque fois du décret d'une grâce, et en retour s'y conformant, Claude Simon s'engage pour sa part dans une voie radicalement opposée en préférant la délibération au hasard, la recherche patiente de la composition au rapt aléatoire, fût-il inespéré. Rien d'étonnant ici. Car, qu’on le veuille ou non, croire pouvoir faire des images à la "sauvette " fleure la pensée magique. Or, on ne trouve rien de ce goût-là chez Simon. La venue de chaque image doit procéder d'un travail, lui-même issu d'une interrogation et d'un tâtonnement, autant dire d'une pensée dégrisée. À cette espèce de chasseur-cueilleur qu'est l'adepte de l' "instant décisif ", il faut par conséquent substituer ici un autre personnage, une sorte de "bricoleur ${ }^{7}$ ", un technicien de la surface des choses visibles, attentif à leurs causes matérielles, habité par une passion d'observer qui saurait, le cas échéant, orienter et infléchir celle de composer. Cadrage il y a bien sûr chez l'un comme chez l'autre, mais c'est en tant que second, essentiellement second, qu'il vaudra chez Simon.

Les choses étant ainsi posées, reste qu'elles ne sont pas simples. Dans la préface qu'il consacre au livre de photographies du romancier, Denis Roche rapporte un échange qu'ils eurent ensemble au sujet de cette affaire du cadrage:

Claude Simon me dit alors: "Quand on fait une photo, on découpe, on tranche dans le temps. Et aussi dans l'espace. On isole (il souligne en parlant) complètement. Alors reca-

6. Hist., p. 324.

7. Le «bricoleur ", comme l'a montré Levi-Strauss, est celui dont la règle " est de toujours s'arranger avec les "moyens du bord", c'est-à-dire un ensemble à chaque instant fini d'outils et de matériaux, hétéroclites au surplus " (La Pensée sauvage [1962], Plon, Pocket, coll. " Agora ", 1990, p. 31). 
drer ne change pas grand-chose à ce qui s'est passé au moment de la prise de vue, on isole un peu plus, c'est tout. " Je lui dis: "En somme, la prise de vue, tu la considères comme une sorte de brouillon, la finition se faisant plus tard par un recadrage et uniquement à ce moment-là? - Absolument, au recadrage, j'enlève ce qui est inutile, je resserre. - Tu fermes l'image? - Oui, c'est ça: je ferme ${ }^{8}$."

L'acte de cadrer, selon Simon, consiste ainsi à éliminer en cernant autrement; cadrer est une façon de sélectionner des éléments, d'épurer l'ensemble pour ne retenir que ce qui participe au sens qui se révèle. C'est à la lettre ce que veut dire "fermer ", qu'on doit entendre comme l'acte d'instituer par le choix d'un contour - champ/hors-champ - l'artifice signifiant d'une image. Bref, on le voit, à chaque moment de son effet et quel qu'en soit l'enjeu local, cadrer, en tant même qu'il consiste à discerner, autrement dit à séparer, est un acte de jugement. Et en l'espèce, juger, c'est détourer. Quant à tenir la prise de vue " comme une sorte de brouillon", cela revient à valider l'analogie que Simon lui-même n'hésite pas à formuler lorsqu'il évoque dans l'avant-propos de ce même livre sa pratique de romancier: "Il y a un point d'équilibre à trouver. C'est à sa recherche difficile que j'écris. Très péniblement, avec beaucoup de ratures. Et c'est à une recherche du même ordre que je me suis livré avec un appareil photographique et une agrandisseuse. "

Ayant gagné en généralité, le problème se trouve désormais exposé dans les termes d'une identité de rapports. Il apparaît ainsi que la mise au point du roman - on songe à ces pages tant de fois retouchées, rehaussées çà et là de couleurs, de croquis - est au matériau initial de l'écriture - à la lettre à son écriture ellemême - ce que le tirage de la photographie, à savoir son cadrage, est à la prise de vue. Ici comme là, il ne s'agit que de revenir sur ses propres pas. Ici comme là tout se règle dans un après-coup par une suite d'amendements (ici, la tâche indéfinie du raturage et de la bifurcation et là, la décision de recadrer pour réformer le regard) jusqu'à obtenir quelque chose (sur une page ou sur du papier sensible) qui donne finalement l'impression d' « à peu près tenir debout ».

Commodité souvent pour la pensée peinant à cerner son objet, il arrive parfois que l'analogie mette au jour ses propres limites. Claude Simon le sait bien:

si je peux enrichir ou parfaire un texte en y insérant ici ou là un mot, une phrase ou même un paragraphe entier, je ne dispose pour corriger mes brouillons photographiques que du choix entre les gradations de divers papiers plus ou moins contrastés et de la possibilité d'y retrancher, jamais d'y ajouter'?

8. D. Roche, «De la ténèbre inverse ", dans C. Simon, Photographies, éd. cit., p. 7.

9. Phot., p. 16. 
L'action de corriger dans les deux cas est alors à ce point de nature différente que des opérations strictement inverses vont la diriger. Autre chose en effet est d'avoir la possibilité de supplémenter, de préciser, de modifier par expansion, autre chose d'être assigné à ce qui se donne, tant et si bien qu'au bout du compte ne reste que le choix d'ôter. En sorte que d'un côté, ne fût-ce qu'en droit, s'offre l'ouverture sur l'infini du repentir, tandis que de l'autre, la clôture du fini s'impose quoi qu'on fasse. Comme si à la mise au net du brouillon du roman correspondait une promesse tandis qu'au tirage de l'image la contrainte seulement. Faudrait-il en conclure que l'analogie en question n'était aux yeux de Claude Simon qu'un expédient? Qu'elle n'était qu'une façon d'évacuer la question de sa double pratique d'écrivain et de photographe? Rien n'interdit de le croire, cela va de soi, sauf à risquer peut-être de manquer le nœud de cette affaire.

Car à bien y regarder, c'est peut-être moins l'analogie elle-même qui doit être suspectée que l'objet sur lequel elle est censée porter. Penser en effet le recadrage en considérant la seule possibilité de l'ajout - ici permis, là impensable - fonctionne à terme comme un piège en laissant de surcroît supposer l'effectivité d'une liberté dans le champ exclusif de l'écriture. En réalité, tout indique qu'en deçà de cette possibilité, l'identité de rapports entre les deux actions - écrire, photographier - subsiste bel et bien, pour peu qu'on s'aperçoive qu'elle se fonde sur la nécessité de la correction et non sur ses modalités. Que Claude Simon ait à travailler sur des "brouillons" dans les deux cas n'implique ni qu'il doive accomplir le même geste ni que l'un soit plus éminent que l'autre. Soit, mais de quel geste parle-t-on ici? Et pour commencer que faut-il entendre par possibilité, voire par nécessité de la correction dans le champ de l'écriture? Dans son introduction au premier tome des Euvres de Claude Simon dans la Bibliothèque de la Pléiade, Alastair B. Duncan cite l'extrait d'un propos publié en 1964 qui peut nous éclairer: "Lorsque j'écris (je m’en rends compte de plus en plus), je n'exprime pas quelque chose qui préexisterait à l'écriture ${ }^{10}$. " Inutile d'y insister, la chose a été plus d'une fois remarquée et commentée: lorsqu'il écrit, Claude Simon tient pour nulle une quelconque possibilité de restitution. À la table, au plus près d'un matériau préalable, en recourant à toutes les modifications qui se présentent, en accueillant toutes les associations et les surprises que réserve le seul acte d'écrire, tout son travail tend à faire advenir une réalité originale dont il n'avait en commençant pas même l'idée: 
où l'aventure commence pour moi, c'est qu'à chaque moment de cette recherche, la langue $[. .$.$] , tout en me forçant de par sa structure linéaire et les lois de sa syntaxe à chercher$ un ordre, désigner des priorités, ne cesse en même temps de me proposer, à chaque mot que je trace, une multitude de perspectives, de chemins possibles, d'images, d'harmonies, d'accords imprévus au départ ${ }^{11}$.

Si bien que la nécessité de correction, c’est-à-dire de reprise, de rectification ou de réagencement, ne consiste jamais à satisfaire à la norme d'un sens univoque, préalable et latent, mais au contraire à admettre à l'existence tout ce que l'inertie de ladite norme aurait toujours déjà forclos. Reste à voir maintenant ce que peut bien recouvrir ce désir de correction lorsqu'on n'a plus affaire au texte en cours d'élaboration mais à l'image telle qu' elle surgit sous la lumière inactinique du labo.

Soit à présent l'une d'entre elles. Le mot Passeur lui sert de titre. On la trouve à la page 115 du livre rassemblant des photographies prises entre 1937 et 1970. Sans tenter réellement d'en faire l'ekphrasis, on dira d'abord qu'elle est en noir et blanc - toutes le sont - et surtout que, malgré le caractère insolite de sa composition, frappe sa sobriété. À la limite supérieure du coin droit, détachée sur un fond gris à peine modulé, une barque de pêcheur, comme happée par la brume. Six à huit personnages y ont pris place. Hormis le premier d'entre eux, légèrement en avant du groupe, côté proue, tous semblent assis ou à peu près. L'homme en question - le passeur? - tient une perche à bout de bras, sorte de gaffe doublant sa propre hauteur, comme s'il sondait, tandis que sur la poupe, un compagnon d'équipage qu'on aperçoit de dos - la tache noire de la tête jure avec celle plus claire d'une vareuse -, manœuvre une longue rame faisant office de gouvernail. Rapportée à la surface tout entière de l'image, on réalise que l'embarcation et ses passagers occuperaient une portion bien modeste de l'espace, relèveraient quasiment de l'anecdote si leur emplacement, on ne peut plus décentré, et les reflets très sombres, à peine chahutés par des vaguelettes, ne révélaient à proportion inverse l'affirmation de leur présence. Ils sont d'autant plus là, d'autant plus essentiels, qu'ils semblent sur le point de disparaître; ou plutôt, qu'ils sont, disparaissant. Mais ce n'est pas tout. La force de l'image tient aussi à ces arguments graphiques que sont à l'avant-poste la verticale de la perche, l'oblique de la rame à l'arrière et, comme surlignant la glissade de la coque, la ligne horizontale d'une flottaison noire. En se combinant, ces trois axes disjoints contribuent à tracer un polygone étrange, lequel, 
posé sur la mer plate d'un jour naissant ou finissant - aucun indice n'est donné -, s'avère d'autant plus fascinant, d'autant plus énigmatique, qu'il dérive en silence - c'est-à-dire sans bouger - vers la zone insensible du hors-champ.

Si cette photo nous retient, c'est évidemment en raison de sa puissance et de sa grande beauté, mais c'est sans doute aussi parce qu'en elle se condense l'ensemble des déterminations qui peuvent dicter de façon générale l'acte de recadrage. Au cours d'un entretien avec Pascale Casanova, Claude Simon avait rappelé combien cette opportunité était pour lui " primordiale ${ }^{12}$ ». Ce qui était d'ailleurs vrai à la table d'écriture l'était a fortiori dans la chambre noire. Cadrer, confiait-il, lui permettait d'isoler "dans l'espace et dans le temps ${ }^{13}$ " les éléments porteurs de sens. Cadrer, en somme, revenait chez lui à coder les flux en les repérant, à organiser le jeu mutuel des signes en fixant leurs rapports au nom $\mathrm{du}$ projet unifiant qu'est toute composition. Pour la photo qui nous occupe, celle dont le titre Passeur donne le ton, ce geste de cadrer, ou plutôt de re-cadrer - on suppose l'effet sensiblement différent de l'image originale -, apparaît vite comme ayant répondu à une volonté précise, celle d'une dramatisation de la scène préalablement vue. Ce qui passait, aux dires mêmes de Claude Simon, pour un geste de second rang, purement soustractif, signe au contraire une intensification d'ordre qualitatif. Preuve que le fait de recadrer, loin d'entamer un sens toujours déjà livré, tend à en produire un nouveau, inattendu et d'un ordre supérieur. Pour en saisir la portée, laissons la place aux conjectures. Imaginons, sans garantie aucune, ce que Claude Simon a choisi de ne pas montrer afin précisément de montrer autre chose. La présence du grain sur l'image, son aspect vaporeux, invitent d'ores et déjà à penser que le recadrage s'est accompagné d'un agrandissement conséquent au moment du tirage. Sûrement est-on passé ici d'un plan assez large - une "marine ", comme on dit en peinture -, donnant à voir une scène convenue, à un cadre beaucoup plus resserré ${ }^{14}$ où allait s'enlever un agrégat sensible, unique et orienté, sur un fond quasi neutre. Cette double opération a certes permis $\mathrm{d}$ ' " isoler » le sujet - «la " barque sur " la » mer -, mais elle a surtout contribué à suggérer une mise en intrigue inédite en surdéterminant les données initiales. De banals pêcheurs qu'ils étaient, tels en tout cas que Simon les avait aperçus à travers son viseur, les silhouettes se sont

12. "Claude Simon. Choses vues ", entretien avec Pascale Casanova, Art Press, n 174, nov. 1992, p. 30-33.

13. Ibid.

14. Le format carré de l'image est trompeur. Il suffit de mesurer les côtés de l'image pour vérifier qu'il s'agit, de très peu, d'un rectangle. Preuve, s'il en fallait, d'une action effective de recadrage, lui-même déterminé ici de manière strictement empirique. 
changées en figures immémoriales. De quasi archétypes: des ombres de contrebandiers ou de clandestins en fuite; peut-être même - et en même temps - des âmes d'humains accomplissant leur ultime voyage.

Sans perdre de vue cette image, essayons pour finir de comprendre quelques enjeux de ce désir du recadrage chez Claude Simon en posant qu'il implique simultanément plusieurs modalités de ce qu'on a nommé en commençant une "éthique du voir ". La première, ne suivant pas ce que Simon lui-même crut devoir avancer, consiste à récuser l'idée d'une quelconque perte en tenant au contraire l'acte de recadrer pour ce qu'il est réellement, à savoir la condition d'un saut qualitatif. En agrandissant, en recadrant, s'accomplit proprement un changement d'échelle. Le système tout entier des proportions, des équilibres s'en trouve redistribué. Haut, bas, proche, lointain, rien ne vaut plus dans l'absolu. Et c'est le sens lui-même qui est rejoué à nouveaux frais. Le rapport entre l'image originale et celle qui a subi le recadrage n'est donc pas celui qui conduit d'un plus au moins ou d'un tout vers la partie. Il atteste en réalité le passage irréversible d'une chose à une autre, si ce n'est à son autre. En ce sens, les photographies pensées, travaillées et montrées par Simon ne sont pas plus des fragments que des variations métonymiques. Elles procèdent de la différence, non de l'amputation. Elles n'existent que sous la condition d'avoir fait droit à la différence elle-même, l'acte de "voir " chez Simon supposant toujours la recherche d'un voir autrement, de voir l'autre chose, celle qui, captive de l'immédiateté d'une perception, ne pouvait qu'échapper.

Évoquer la deuxième modalité invite à suivre maintenant le chemin de pensée qui accompagne le recadrage lui-même. Ce chemin, analogue à celui qu'emprunte l'écriture du roman, se trouve tout à la fois à partir de visées et de tâtonnements. Il se trace au fur et mesure, s'affine ou s'épaissit à la faveur d'approximations et de rectifications successives. On imagine ici Claude Simon, porté par une intuition, penché sur l'image en devenir, faisant glisser son support dans un quadrilatère de lumière crue, modifiant peu à peu son format, rêvant l'imminence d'un sens. On l'aperçoit, pensant devoir supprimer telle ou telle partie de l'image tandis que conspire déjà ce qu'il retient à instaurer l'existence d'un monde inédit. On se souvient alors de ce passage du Philèbe, de ces personnages en train de converser à propos d'une forme qu'ils viennent d'apercevoir au loin et qu'ils tentent, chemin faisant, de définir: "- Qu'est-ce que c'est? - C'est un homme. - Non, ce n'est pas un homme. - C'est une statue taillée par un berger. » Et Platon de nous rappeler ici encore que la pensée agit de même en 
notre âme, en empruntant l'allure d'un dialogue silencieux qui, en s'exposant, se cherche et s'affecte; ajoutant pour finir au sujet de l'âme qu' " elle ressemble alors à un livre ${ }^{15}$ ". C'est une enquête réflexive identique, au plus près du réel auquel elle a affaire et qu'elle tente d'approcher, qu'actualise l'écriture de Claude Simon. Pour les avoir lus maintes fois, on connait quelques-uns des marqueurs qu'elle sait fréquemment mobiliser; tous ces "non pas ", ces "plutôt " (pondérés par des « de même que »), lesquels, en distinguant, en précisant ou corrigeant - comme sous l'agrandisseur -, soutiennent le mouvement de la phrase pensive, l'énergie de son phrasé aussi bien, et participent surtout à la mise au jour progressive de dimensions ontologiques jusque-là inaperçues. Deviennent sensibles ici non pas de banals détails - une barque sur la mer -, mais le foisonnement infini d'existences mineures qu'une saisie totalisante ne pouvait, de prime abord, qu'offusquer. Tout cela pour ne pas oublier que de l'immédiat ne se donne jamais que le rien: " et peut-être ne voyant même pas, pas plus que l'appareil photographique lui-même ne voit, ne connaît, n'est capable de se souvenir ${ }^{16}$ "; que nous devons par conséquent nous employer à re-voir pour voir peut-être enfin.

Qu'il intéresse l'élaboration du roman ou la mise au net d'une image, cet acte de re-voir coïncide avec celui de corriger, de rénover notre cadre d'appréhension des choses. N'impliquant en aucun cas un mieux-voir, cet acte ne se nourrit en vérité que d'un désir: composer par la fiction quelque chose qui puisse offrir ce qui n'était pas encore visible. Non pas un "comment c'était "; plutôt un comment-ça-pourrait-être: "J'étais assis sur mon divan, la fenêtre ouverte, un pigeon est passé entre le soleil et moi. J'ai écrit ça en me disant: on va voir ce qui va venir ${ }^{17} \ldots »$

15. Platon, Philèbe, tr. A. Diès, Les Belles Lettres, 1959, $38^{\mathrm{e}}$.

16. Le Vent, p. 36.

17. C. Simon, "Attaques et stimuli", entretien dans L. Dällenbach, Claude Simon, Le Seuil, coll. "Les contemporains », 1988, p. 171. 